\title{
THE SOLAR-RADIATION PRESSURE EFFECTS ON THE ORBITAL EVOLUTION OF ASTEROID MOONS
}

\author{
V.V. Troianskyi, O.A. Bazyey \\ Astronomical Observatory, Odessa National University \\ Shevchenko Park, Odessa 65014, Ukraine \\ v.troianskyi@onu.edu.ua,o.bazyey@onu.edu.ua
}

ABSTRACT. In the theory of motion, disturbances are divided into gravitational and non-gravitational ones. In this paper, we discuss the effects of solar-radiation pressure on the orbital evolution of asteroid moons.

It is known from the laws of physics that the smaller an object is the more pressure is exerted on it by solar radiation. That is the reason why asteroid moons with their small sizes are exposed to the solar-radiation pressure so much.

Key words: asteroid, asteroid moons, orbital evolution.

\section{Equation of motion}

To improve the accuracy of the theory of motion of asteroid moons, it is necessary to account for the effects of the solar-radiation pressure on their surfaces. For this purpose, the acceleration due to the solar-radiation pressure was expanded (Duboshin, 1971):

$$
\begin{aligned}
& a_{L p x}=(1+k) q \frac{S}{m} \Psi\left(\frac{a}{r_{0}}\right) \frac{x_{S t}-x_{S}}{r_{0}} \\
& a_{L p y}=(1+k) q \frac{S}{m} \Psi\left(\frac{a}{r_{0}}\right) \frac{y_{S t}-y_{S}}{r_{0}}, \\
& a_{L p z}=(1+k) q \frac{S}{m} \Psi\left(\frac{a}{r_{0}}\right) \frac{z_{S t}-z_{S}}{r_{0}} \\
& r_{0}=\sqrt{\left(x_{S}-x_{A}\right)^{2}+\left(y_{S}-y_{A}\right)^{2}+\left(z_{S}-z_{A}\right)^{2}},
\end{aligned}
$$

where $k$ - the albedo; $q=4,5605 \cdot 10^{-6} \frac{N}{m^{2}}$ - the solar constant; $S$ - the cross-sectional area of the asteroid moon; $m$ - the asteroid moons' mass; $\Psi$ - the shadow function; $\alpha$ - the mean distance from the asteroid to the Sun; $x_{A}, y_{A}, z_{A}$ - the asteroid's coordinates; $x_{S}, y_{S}, z_{S}-$ the Sun's coordinates; $x_{S t}, y_{S t}, z_{S t}$ - the asteroid moon's coordinates.

As a result the differential equations describing the motion of the asteroid moon in the asteroid-centric inertial coordinate frame can be formularized as follows:

$$
\begin{aligned}
& \frac{d^{2} x}{d t^{2}}-a_{L p x}=0 \\
& \frac{d^{2} y}{d t^{2}}-a_{L p y}=0 \\
& \frac{d^{2} z}{d t^{2}}-a_{L p z}=0
\end{aligned}
$$

where $x, y, z$ - the position vector components; $t$ - time; $a_{L p}-$ the acceleration due to the solar-radiation pressure.

To integrate differential equations of motion, the $15^{\text {th }}$ order Everhart method was employed (Bazyey et al., 2009).

\section{Shadow function}

The conception of the shadow function $\Psi$ was suggested by Ferraz-Mello in 1963 (Duboshin, 1971). This function equals to 1 when the natural satellite is illuminated by the Sun, and it is zero when the moon is in the shadow. At a first approximation, the shadow is reckoned to be cylindrical-shaped; but when higher accuracy is required, it is assumed that the shadow is cone-shaped.

Let us set the shadow function as $\Psi$. Then, for the cylindrical-shaped shadow we obtain the following:

$$
\Psi=\left\{\begin{array}{l}
0,-\varphi<\lambda<\varphi, \\
1,-\pi<\lambda<-\varphi, \varphi<\lambda<\pi .
\end{array},\right.
$$

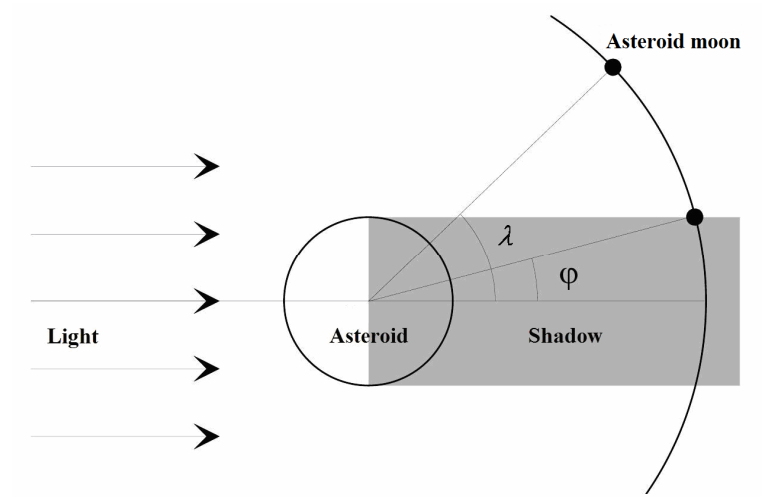

Figure 1: Geometric representation of the cylindrical-shaped shadow. 


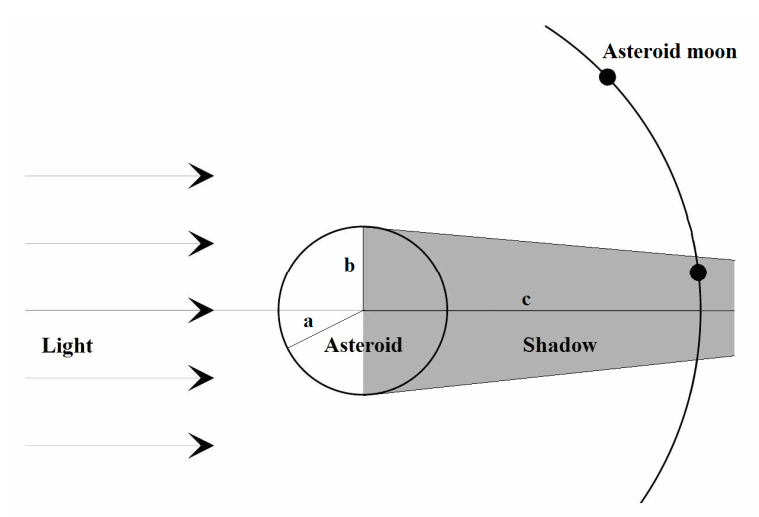

Figure 2: Geometric representation of the cone-shaped shadow.

In our model the shadow is supposed to be represented as cone-shaped; that enables to determine more accurately the instants when an asteroid moon enters into and exits from the shadow.

$$
\Psi=\left\{\begin{array}{l}
0, \frac{x_{S}^{2}}{a^{2}}+\frac{y_{S}^{2}}{b^{2}}-\frac{z_{S}^{2}}{c^{2}}=0, \\
1, \frac{x_{S}^{2}}{a^{2}}+\frac{y_{S}^{2}}{b^{2}}-\frac{z_{S}^{2}}{c^{2}}=1 .
\end{array},\right.
$$

where $a, b, c$ - the cone axes. The compression of the body casting a shadow is also accounted for the shadow shape.

\section{Orbital evolution of asteroid moons}

As an example, we integrated the equations of motion of the moon of asteroid (66391) 1999 KW4 (http://www.johnstonsarchive.net/astro/astmoons/am-66391. html) over 1000-year interval accounting for the shadow function. The integration step is 6 minutes with the integration error $10^{-7} \mathrm{~m}$.

As can be seen from the results obtained, only orbital inclination and the ascending node longitude have a secular component of the disturbance by the acceleration due to the solar-radiation pressure. The orbital inclination changes by 1 degree every 900 years while the longitude of the ascending node changes by 0.5 degree every 75 years. That can be also seen in Figs. 3 and 4.

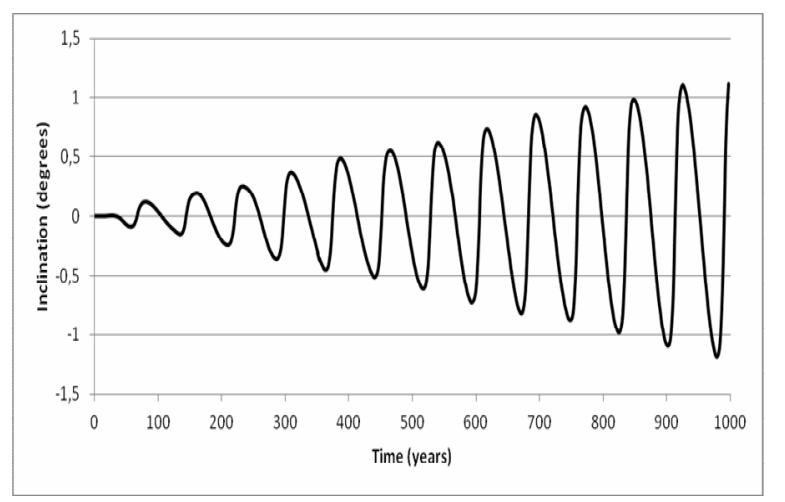

Figure 3: Changes in the orbital inclination of natural satellite Beta in asteroid system (66391)1999KW4.

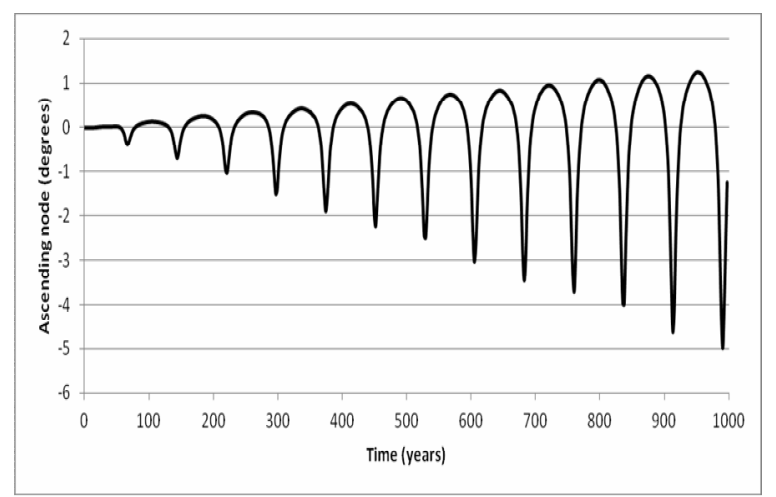

Figure 4: Changes in the longitude of ascending node of natural satellite Beta in asteroid system (66391) 1999KW4.

\section{Results}

The obtained results show that the orbital inclination and longitude of the ascending node increase secularly; hence, it can be concluded that the acceleration due to the solar-radiation pressure is one of the reasons why asteroid moons go into retrograde orbits. For instance, the orbital inclination of the moons in asteroid systems (22)Kalliope (Margot et al., 2003), (93)Minerva (Marchis et al., 2013), (66391)1999KW4 (Ostro el al., 2006) and (153591)2001 SN263 (Fang et al., 2001) exceeds $90^{\circ}$.

To sustain this hypothesis, further more detailed investigation of the indicated asteroid systems over a longer interval of time is required.

\section{References}

Bazyey A.A., Kara I.V.: 2009, Visnyk Astronomichnoyi shkoly, 6/2, 155 (in Ukrainian).

Duboshin G.N.: 1971, Reference Guide on Celestial Mechanics and Astrodynamics.

Fang J., Margot J.-L., Brozovic M., Nolan M.C., Benner L.A.M., Taylor P.A.: 2011, Astron. J., 141, 154.

Margot J.L., Brown M.E.: 2003, Science, 300, 1939.

Marchis F. et al.: 2013, Icarus, 224, 178.

Ostro S.J. et al.: 2006, Science, 314, 1276. 